\title{
Specimen slice radiography of cancer in breast conserving excisions
}

\author{
K L Oakley, J J Going
}

\begin{abstract}
Aim-To investigate histologically the power of specimen slice radiography to record invasive and in situ carcinoma in breast conserving cancer excisions.

Methods-Twenty six cancer excisions were converted into 171 complete tissue slices, which were examined radiographically. From these slices, 373 histological blocks were processed and histological and radiographic assessments were compared.

Results-Radiography and histology mapped excision margins and lesions in detail. Radiographic prediction of histology was imprecise. Six invasive carcinomas were either undetectable by radiology or reached a radiologically clear margin. Six small invasive carcinoma satellites were not recognised. Adjacent ductal carcinoma in situ was undetectable in nine of 15 cases.

Conclusions-Slice radiography and histology are capable of precise lesion mapping in breast cancer excisions, and clinical utility of such mapping merits investigation. Radiology alone is imprecise and to infer complete excision of breast carcinoma by radiography (of excision specimens or residual breast) alone may be unsafe.

(f Clin Pathol 1995;48:1028-1030)
\end{abstract}

Keywords: Breast carcinoma, ductal carcinoma in situ, radiography.
Multifocality, adequate excision, and the distribution and type of associated carcinoma in situ $^{12}$ are significant in breast conserving cancer surgery. The aim of this study was to investigate the potential of specimen slice radiography to yield useful information about these matters during and after breast conserving surgery for symptomatic cancer.

\section{Methods}

Twenty six unfixed breast conserving cancer excisions were sliced ${ }^{3}$ into three to 13 (median, seven) serial sections, $4 \mathrm{~mm}$ thick (fig 1 ). Contact radiography of slices was carried out on Cronex mammographic film (DuPont) without an intensifying screen $(20 \mathrm{kV}, 60$ seconds). Unfixed tissue for frozen section histology, receptor assay and tissue banking was then taken without disturbing resection margins. Slices were fixed overnight in $10 \%$ neutral buffered formalin between sheets of blotting paper. Between four and 27 tissue blocks (median, 12) were processed for histology per case. Blocks chosen were marked on the radiographs, which were assessed for the distribution of invasive and in situ carcinoma; correctness of these judgements was verified histologically. Most resection margins were inked after slicing and fixation, but the margins of some unsliced lumpectomy specimens were dyed by immersion in $1 \%$ aqueous alcian blue. These specimens were washed in tap water and dried before slicing.

\section{Results}

Satisfactory slicing and clear radiographic images were obtained (fig 2). Alcian blue staining before slicing made occasional slicing errors obvious. Invasive carcinoma was confirmed by histology in all specimens and was identifiable from abnormal architecture, with or without microcalcification, in radiographs of 22 cases. Invasive carcinoma was thought to reach the radiographic resection margins in three cases; this was confirmed histologically. The margins were not involved in the three cases with ambiguous radiography. Invasive carcinoma reached radiographically clear margins in three of 20 cases.

Distinctly separate satellite foci of invasive carcinoma in eight cases were detected by radiography in two. All of these satellites were small (1-2 mm). Satellites suspected on radiography in four cases were confirmed in two. Ductal carcinoma in situ outside the dominant focus of invasive carcinoma in 15 cases was confidently identified on slice radiography in
Figure 1 Slicing a lumpectomy. Six slices have been made and a seventh is being taken. The hand holding the specimen is protected by chain-mail and latex gloves. 


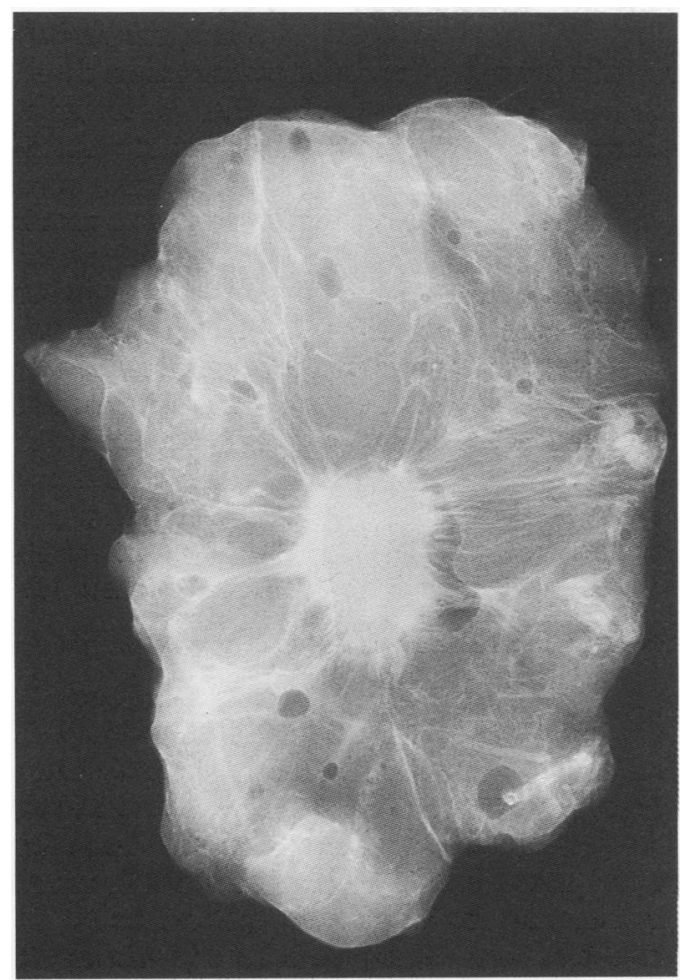

Figure 2 Lumpectomy slice radiograph. The structure of the slice and centrally placed $14 \mathrm{~mm}$ carcinoma are recorded in detail. The slice margins are excision margins.

four. One confident prediction of ductal carcinoma in situ was refuted on histology, and two of four cases with radiographs suggestive of ductal carcinoma in situ were confirmed histologically. Nine ductal carcinoma in situ areas were not detected on radiography, and could not be detected on slice radiographs, even in retrospect.

\section{Discussion}

A desire for breast cancer mapping, particularly near resection margins, as part of breast conserving cancer treatment is indicated by the use of cavity shaving and tumour bed biopsy after initial lumpectomy. Two groups advocate these procedures, ${ }^{4-8}$ and clearly they do not think standard pathological prosection and reporting of lumpectomies provide adequate information. Perhaps they are right, but it is possible that these procedures do not yield information that could not be derived from an intact lumpectomy, carefully orientated and scrupulously examined, given the logical equivalence between a number of portions of breast tissue extracted from a single biopsy cavity, and the same volume of breast tissue excised as a single lumpectomy, from which histological blocks equivalent to cavity shavings and tumour bed biopsy specimens could be derived. The approach described by England $e t a l^{9}$ is different, as they used surface scraping cytology as an adjunct to tumour bed biopsy. If the purpose of cavity shaving and bed biopsy is to enforce separate histological reporting of multiple blocks from resection margins, because pathologists fail to do so, this should give some cause for concern.
Slice radiography records the structure of breast conserving cancer excisions in detail, and allows tissue blocks, lesions and marginal relations to be visualised in three dimensions. Specimen orientation relates this three dimensional map to the residual breast. Specimen slice radiography therefore seems to be a possible vehicle for accurate pathological reporting of lumpectomy specimens, and its clinical application merits further study. Oncology teams should be aware that such mapping can be achieved in a surgical pathology laboratory with facilities for specimen radiography and the necessary human resources. Slicing unfixed breast tissue accurately requires skill and practice, and is best done by a small cadre of laboratory staff. Mastectomy studies by Holland $e t a l^{10}$ have shown that a substantial minority of patients would have residual invasive or in situ carcinoma even when the principal focus of carcinoma is excised with a substantial margin. It is possible that more detailed cancer mapping in lumpectomies could help to predict this situation.

Without histology, specimen slice radiography is not especially helpful. In the present study resection margins were clearly visualised by radiography (except in the polar slices), optimising conditions for recognising inadequate excision, but specimen slice radiography did not yield sufficiently accurate intra-operative data concerning the integrity of resection margins to guide further surgery (immediate or delayed) without histological confirmation.

The frequency with which ductal carcinoma in situ was undetectable, even in hindsight, in specimen slice radiographs must challenge the recent assertion that careful mammography may reliably assess the extent of ductal carcinoma in situ, and thus ensure its complete excision. ${ }^{11}$ It is possible that the sensitivity with which ductal carcinoma in situ is detected by radiography may depend upon its histological type. This is suggested in another study by Holland $e t a l,{ }^{12}$ which demonstrated a greater discrepancy between mammographic and pathological size for non-comedo than for comedo ductal carcinoma in situ subtypes. The present series includes too few cases for definitive appraisal of this possible relation. Ductal carcinoma in situ not detected on slice radiographs is unlikely to be detected in the more complex radiographic environment of the breast.

The specimen radiography cabinet was bought with a grant from the Scottish Home and Health Department.

1 Connolly JL, Schmitt S. Evaluation of breast biopsy specimens in patients considered for treatment by conservative surgery and radiation therapy for early breast cancer. Pathol Annu 1988;23:1-23.

Sloan JP. Changing role of the pathologist. Br Med Bull 1991;47:433-59.

3 Anderson TJ. Breast cancer screening: Principles and practicalities for histopathologists. In: Anthony PP, MacSween RNM, eds. Recent advances in histopathology. Vol 14. EdinRNM, eds. Recent advances in histopathology.

burgh: Churchill Livingstone, 1989:43-61.
4 Umpleby HC, Herbert A, Royle GT, Taylor I. Wide excision of primary breast cancer: the incidence of residual carof primary breast cancer: the incidence of residual car-
cinoma at the site of excision. Ann R Coll Surg Engl 1988; cinoma at the

5 Carpenter R, Cross M, Herbert A, Royle GT, Taylor I Tumour bed biopsy: a new prognostic indicator following conservative treatment of early breast cancer [abstract] Br F Surg 1989;76:1334. 
6 Macmillan RD, Purushotham AD, George WD. Breast conserving surgery and tumour bed positivity in patients with breast cancer. $B r \mathcal{F}$ Surg 1994;81:56-8.

7 Taylor I, Carpenter R, Royle GT. Breast-conserving surgery and tumour bed positivity in patients with breast cancer [abstract]. Br $\mathcal{F}$ Surg 1994;81:922.

8 Purushotham AD, Macmillan RD, George WD. Breastconserving surgery and tumour bed positivity in patients with breast cancer. Br f Surg 1994;81:922-3.

9 England DW, Chan SY, Stonelake PS, Lee MJR. Assessment of excision margins following wide local excision for breast carcinoma using specimen scrape cytology and tumour bed biopsy. Eur $\mathcal{f}$ Surg Oncol 1994;20:425-9.

10 Holland R, Veling SHJ, Mravunac M, Hendriks JHCL. Histologic multifocality of Tis, T1-2 breast carcinomas: implications for clinical trials of breast conserving cancer surgery. Cancer 1985;56:979-90.

11 Recht A, van Dongen JA, Peterse JL. Ductal carcinoma in situ [abstract]. Lancet 1994;343:969.

12 Holland R, Hendriks JHCL, Verbeek ALM, Mravunac M Schuurmans S. Extent, distribution, and mammographic/
Schand histological correlations of breast ductal carcinoma in situ. Lancet 1990;335:519-22.

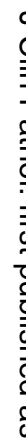

